\title{
Multi-Symmetric Level (MSL) Optimization Technique Based on Genetic Algorithm for Photonic Devices Design
}

\author{
Marcos S. Gonçalves $^{1}\left(\mathbb{D}\right.$, Carlos H. Silva-Santos ${ }^{2}$ (D) Wilton M. Ferraz-Júnior ${ }^{1,2}$ (D), \\ Francisco J. Arnold ${ }^{2}$ (D) \\ ${ }^{1}$ School of Technology, University of Campinas (Unicamp), Limeira 13484-332 1888, Brasil, \\ marsergo@unicamp.br,arnold@unicamp.br \\ ${ }^{2}$ Federal Institute of Education, Science and Technology of São Paulo (IFSP), campus Itapetininga, Itapetininga \\ 18202-000 1561,Brazil,carlos.santo@ifsp.edu.br,wiltonjr@ifsp.edu.br
}

\begin{abstract}
The optimization of three-dimensional device has widely been explored and designated as computational challenge in photonic device design. In this context, this work presents a novel multi-symmetric level (MSL) optimization procedure based on Genetic Algorithm (GA) to decrease the computational requirements in a three-dimensional photonic coupler device optimization. The proposed procedure divides the device optimization into many local regions by dimensional symmetric aspects, being based on divideto-conquer strategies. As the optimization converges to acceptance values, the number of symmetric areas are decreasing until a unique local area that modeled the total 3D photonic device. This procedure was validated by the minimization of a photonic coupler modeled to interconnect optical waveguides with different height, width and refractive indices.
\end{abstract}

Index Terms - 3D Optical Coupler, Finite Element Method (FEM), Genetic Algorithm, Numerical Modeling

\section{INTRODUCTION}

The computational and telecommunications data processing and store have worldwide challenged for the design of novel devices [1]. This is usually started by the modelling and simulation of those three-dimensional devices under certain application and fabrication conditions [2]. It also occurs in highcontrast based on index material to scale down ultra-compact optical devices for photonic integrated chip (PIC) for optical communications. Thus, it is mainly motivated by light controlling propagation techniques on small regions by photonic band-gap (PBG) with photonic crystal structures that have been widely explored [3], [4]. Photonic crystals are manufactured periodic structures designed to exhibit photonic band gap [5]. Thenceforth, the crystal geometrical variations have worldwide been explored in different contents [6], such as novel antennas [7]-[9], coupling [10], [11] and filtering devices [12], [13].

Another class of geometries used in device design is metamaterials, in which subwavelength periodic structures are build to design devices with non-conventional physics properties, such as time varying electromagnetic fields with left-handed refractive conditions [14]. Therefore, metamaterial is usually defined to provide alternative properties, such as negative refractive index and thermal expansion coefficients mainly motivated by the complex design of novel 3D metamaterial. The design of 3D metamaterials have been initiated around 20 years ago motivated by those physical contributions and 
recent developed computational aided design and high performance computer approaches to proceed simulations and optimizations in their geometrical properties [15].

However, by the complexity and the number of attributes to be considered in these simulations, a high performance computational approaches and sophisticated numerical alternative techniques are requirements for these executions [16]. It is an affirmation subsidized by the dual challenge regarding the devices miniaturization and expected performance in the application [17]. As an example, photonic couplers modelling that are widely used as integrative devices in circuits designed in nano and micrometer scale circuits [18].

These computational requirements are increased when artificial intelligence metaheuristics based on nature-inspired concepts are integrated in these numerical modelling simulations to optimize these devices parameters [19]. As examples, considering bi-dimensional optimizations where devices are designed applying subwavelength lattice optics [20] and photonic band gap structures optimizations [21], [22], as well as for new optical devices design, [23], beam splitters [24], optical power couplers [24], [25], [26], waveguide filters [27] and optical circuit designed by plasmonic nano rods [28] are some of these recent applications.

Due to the optimization techniques and computational power required in three-dimensional (3D) photonic modeling, the development of novel photonic devices are multidisciplinary afforded challenges considered in this work. In this context, this work aims to present an alternative methodology to reduce the computational costs by partitioning the 3D model of a photonic device into some two-dimensional regions of the photonic device that will regroup as satisfactory solutions are found. By the last step, an optimization with fewer iterations is performed in the three-dimensional model to return its final configuration. This multilevel optimization is based on the divided-to-conquer concept [29], considering device geometry symmetry along the optimized steps that is performed by a Genetic Algorithm.

This method has been validated in the design of a novel minimized 3D optical coupler with $4.2 \mu \mathrm{m}$ of length, following the 2D concept presented in [25]. However, this 3D model take into account optical waveguides with different height and width and the results show that the efficiency is greater than $90 \%$ in wavelength region of $1.55 \mu \mathrm{m}$, with is composed of 1260 cylinders in the coupler region, where each of these cylinders were considered as input optimization unknown to be combined. In second section is presented the photonic coupler modeled and some brief description about Finite Element Method used in this article. The section three presents the optimization method based on the divided-to-conquer to randomly select the refractive index of each of them to maximize the output coupling. The results are presented in fourth section, followed by final considerations and work in progress in fifth section.

\section{The Optical Coupler Architecture}

The photonic device used to validate the proposed method, as already mentioned, is a coupler device with different input and output waveguide dimensions, as shown Fig. 1, and it is based on 2D coupler shown in [24], [25]. The coupler is modeled by a three-dimensional vector finite element method (3DVFEM) in frequency domain developed to this application [30].

The Fig. 2 presents the three-dimensional model of the optical coupler with input waveguide refractive index $n=1.98$ and $2 \mu \mathrm{m}$ width, $1 \mu \mathrm{m}$ height, and $1.5 \mu \mathrm{m}$ length. The output waveguide has core with a refractive index of 3.5 and dimensions of $0.2 \mu \mathrm{m}$ and $0.3 \mu \mathrm{m}$ for height and width, respectively, and its length is $4 \mu \mathrm{m}$. This length is longer than the input waveguide in order to allow calculating 


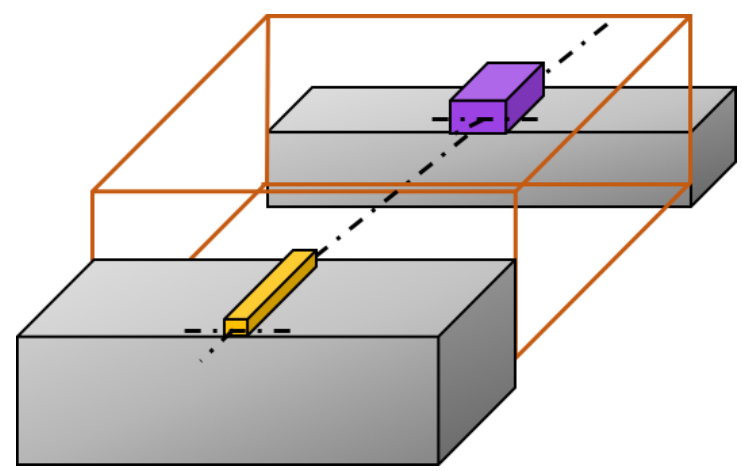

Fig. 1. General 3D Photonic Coupler Schematic.

the modes coupled far from the coupler structure. It was performed to avoid the interference of the non-coupled fields in the final results. The Fig. 2 shows the input and output waveguides in detail.

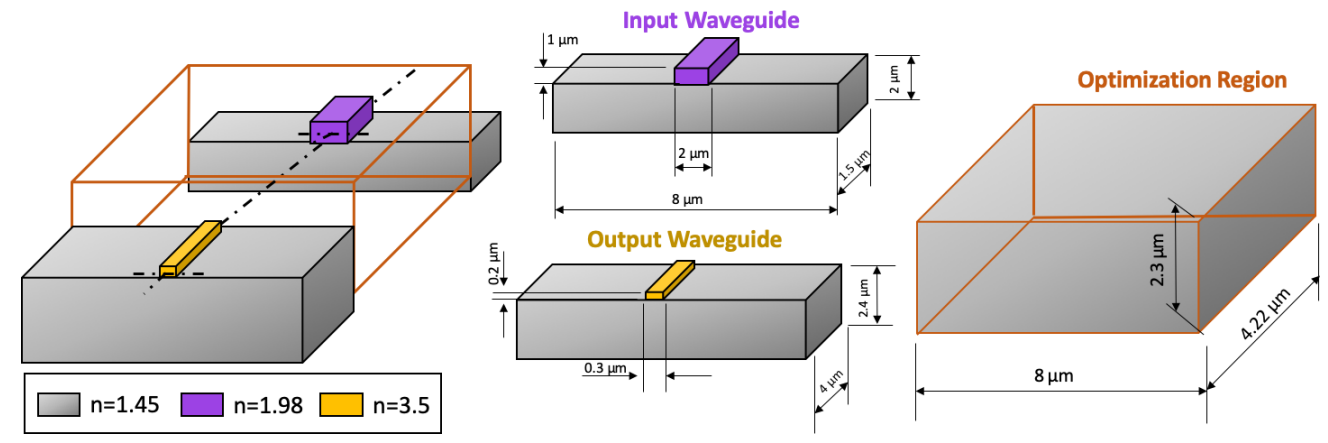

Fig. 2. Photonic coupler device properties.

The optimization region is the place where GA will execute random changes on equally distributed and juxtaposed internally 1260 cylindrical segments distributed in 12 segments in width (Fig. 3), 15 in length and 7 in height. Each one has diameter of $0.2 \mu \mathrm{m}, 0.125 \mu \mathrm{m}$ of height and its periodicity is $0.35 \mu \mathrm{m}$ horizontally and $0.325 \mu \mathrm{m}$ vertically. Henceforth, this optimization region considers $8 \mu \mathrm{m}$ width, $2.3 \mu \mathrm{m}$ height, and $4.22 \mu \mathrm{m}$ length. The optimization region is a box made of a material with 1.45 refractive index.

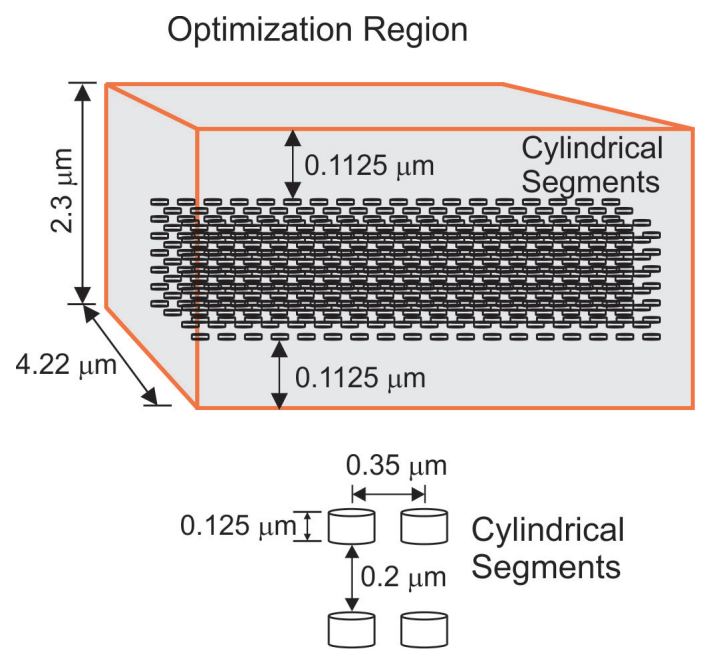

Fig. 3. Optimization region.

Brazilian Microwave and Optoelectronics Society-SBMO Brazilian Society of Electromagnetism-SBMag received 11 May 2021; for review 21 May 2021; accepted 10 Aug 2021 (C) 2021 SBMO/SBMag (cc) BY 
Considering the total number of 1260 cylinders in the optimization region and the Multi-Symmetric Level GA System by directional geometrical simplification, at first the total 1260 is divided by four elements to be recombined to search for suitable solution, which means the combination between 315 cylinders are necessary at first optimization level. From this first step, the second level consider half number of 1260 elements to be combined, which summed 630 cylinders to be combined for an acceptable configuration under the objective functions specifications. Finally, considering the previous configurations as initial start point, the entire 1260 cylinders are considered to search for best solutions to attempt the device design configuration, herein a three-dimensional photonic coupler is considered.

Thenceforth, the total number of cylindrical segments defines the unknowns that will be optimized by the GA, which means 1260 cylinders used in this three-dimensional model. Furthermore, each segment can assume three different refractive index values. However, the computational effort required is too high for attempting all possibilities. As consequence, the optimization process did not converge to a satisfactory result in initial tests, motivating the previously proposed optimization procedure by the association of multilevel symmetric application and Genetic Algorithm.

The device is surrounded by Perfectly Matched Layers (PML) with $1 \mu \mathrm{m}$ thickness and the whole computational domain was discretized in 369514 tetrahedral elements generating 412499 field values. The fundamental mode of the electric field was applied in the input waveguide in the wavelength of $1.55 \mu \mathrm{m}$.

The 3D-VFEM is equated from the wave equation in frequency domain given by (1), where $\Phi=$ $H_{x} \hat{x}+H_{y} \hat{y}+H_{z} \hat{z}$ in the magnetic field and $\Phi=E_{x} \hat{x}+E_{y} \hat{y}+E_{z} \hat{z}$ [31]. The $p=1 / n^{2}$ and $q=1$ in magnetic and $p=1$ and $q=n^{2}$ in electric fields, respectively, where $n$ is the refractive index and $k_{0}$ is the wavenumber.

$$
\nabla \times p \nabla \times \Phi(r)-q k_{0}^{2} s^{2} \Phi(r)=0
$$

The Perfeclty Matched Layer (PML) is introduced by $s$ parameter, where in PML region is defined by (2), where $\omega_{0}$ is the angular frequency, $d$ the PML thickness, and $R$ the theoretical reflection coefficient and $\rho$ is the distance from an internal PML element to the last field simulation and $\nabla$ is modified nabla operator [32].

$$
1-j \frac{3 c^{2}}{2 \omega_{o} n d}\left(\frac{\rho}{d}\right)^{2} \ln \left(\frac{1}{R}\right)
$$

The 3D-VFEM could be described by $\Omega$ computational domain after the Galerkin method application by (3), where $W$ is the Whitney edge base function and $D_{1}$ and $D_{2}$ is given by (4) and (5), respectively, and $\Phi_{i n}$ is the incident field [31].

$$
\begin{gathered}
\iiint_{V e} p \nabla \times \Phi \cdot \nabla \times W_{i} d V-\iiint_{V_{e}} q k_{0}^{2} s^{2} \Phi \cdot W_{i} d V=\iint_{\Gamma_{e}} p\left[D_{1}+D_{2}\right] d s \\
D_{1}=-W_{i_{x}}\left(-j \beta\left(\Phi_{i n, x 2}+\Phi_{i n, x 1}\right)+\left(\frac{\partial \Phi_{i n, z 2}}{\partial x}-\frac{\partial \Phi_{i n, z 1}}{\partial x}\right)\right. \\
D_{2}=-W_{i_{y}}\left(-j \beta\left(\Phi_{i n, y 2}+\Phi_{i n, y y}\right)+\left(\frac{\partial \Phi_{i n, z 2}}{\partial y}-\frac{\partial \Phi_{i n, z 1}}{\partial y}\right.\right.
\end{gathered}
$$

This numerical method was implemented in Fortran 95 language to attempt the computational high 
performance required by the matrix inversion, the ScaLAPACK ${ }^{1}$ was integrated for parallel processing. It was available a computer cluster with 5 nodes, each one with Intel i5 processor $(3 \mathrm{GHz})$ and $16 \mathrm{~GB}$ of RAM memory. The 3D-VFEM execution under these computational and numerical conditions spent around 114 seconds, in average, for one GA iteration.

\section{The Optimization Procedure}

Following Photonic Crystal and Metamaterial challenges to optimize their geometric design, this work aims to present a novel methodology, so-called Multi-Symmetric Level (MSL), to explore symmetric physical properties of a pre-established geometry. In this method, the number of elements to be optimized increases as the results improve. A multisymmetric level is based on multiple executions of a metaheuristic algorithm, the classic Genetic Algorithm (GA) was chosen, and it is an iterative procedure considering the symmetric properties of the device in one and two directions (axis). To increase the number of unknowns, here associated to the refractive index, the geometry have their values customized by the GA throughout the iterations.

The MSL is represented in Fig. 4, where the first GA execution is named by "Genetic Algorithm $(I)$ ". The unknowns number is a quarter of the total parameters pre-established for the device, being determined by four quadrants that divide the device in four equal regions (I, II, III and IV). Hence, the best configuration reached is reused to generate the initial population of the next GA (Genetic Algorithm (II) execution, which decrease the symmetric configurations only for two regions (I and II). The second execution generates best solutions whose configurations will populate the final GA execution, herein nominated by Genetic Algorithm (III), whose results returned the best device configuration. Finally, the last execution level is the freely three-dimensional optimization, considering the previously symmetric optimization results as initial choices.

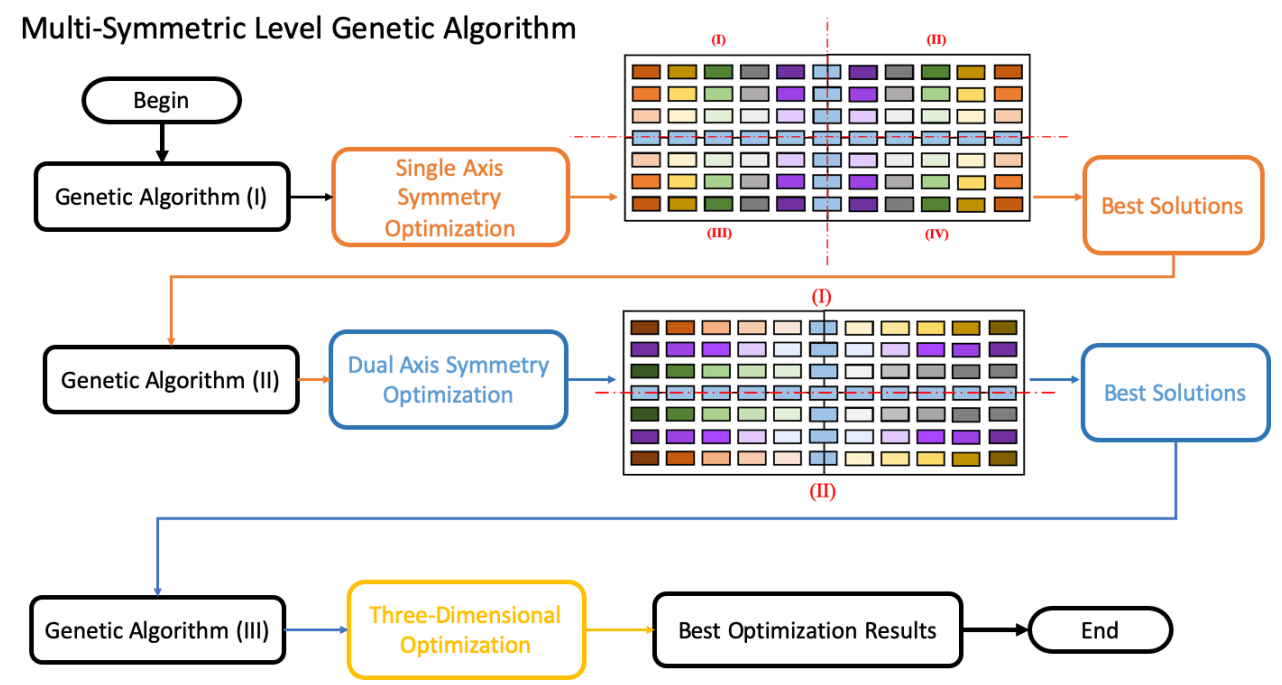

Fig. 4. Main MSL Genetic Algorithm dataflow.

The Genetic Algorithm (GA) is structured by an integer gene mimetization, which main dataflow started with random population generation, whose each candidate solutions, herein called chromosome by GA, and nInd parameter is associated to the number of individuals in the population. Each candidate

${ }^{1}$ http://www.netlib.org/scalapack/ 
solution is calculated by a objective function (fitness) [33]. This general data-flow is presented in Fig. 5 , which is followed by a stop criteria condition to control the iterative procedure of this meta-heuristic. In this work the fitness is designated by 3D-VFEM method for the photonic device modeling and stop criteria is the maximum iteration number of the GA, which is called generations.

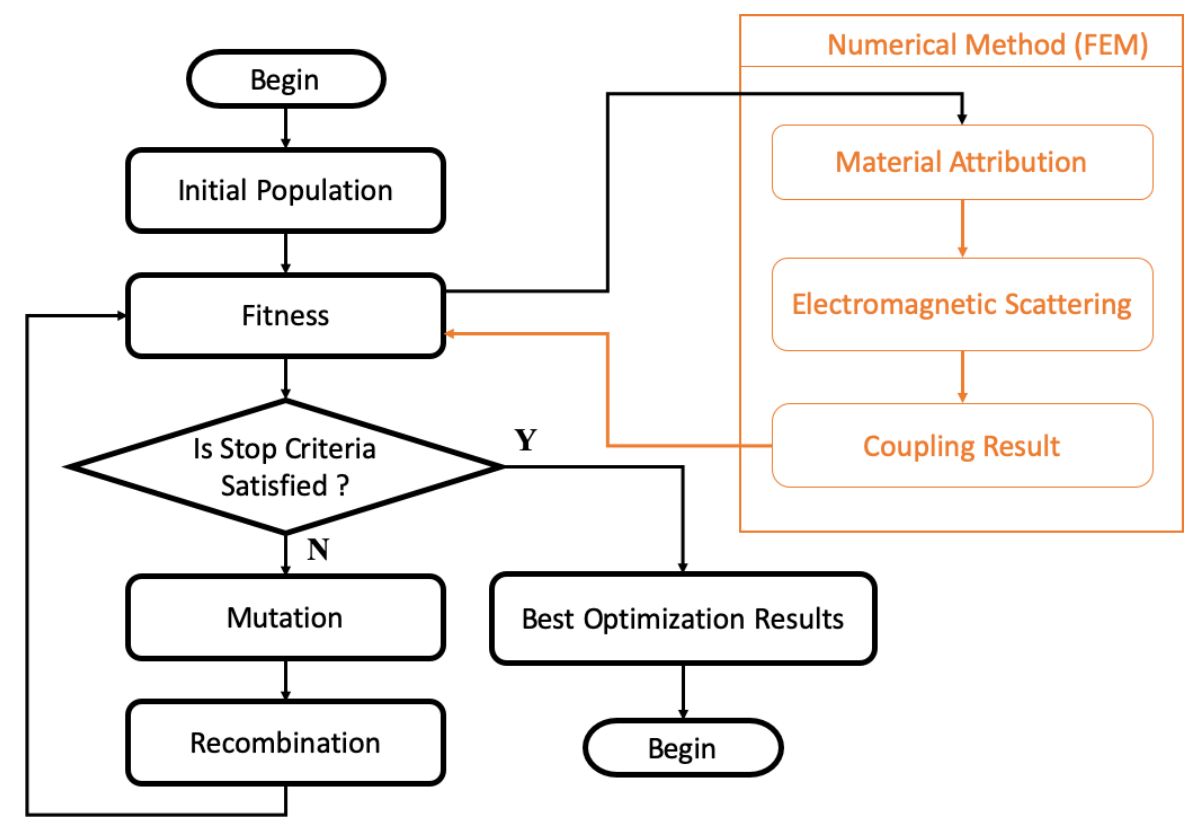

Fig. 5. Main Genetic Algorithm dataflow.

Therefore, the GA population is described by the matrix $P$ in (6), where each chromosome is a vector with $U$ unknowns that are randomly changed by GA operators known for mutation and crossover [34]. Basically, the mutation operator is a uniform random function that randomly choose any element (gene) of the chromosome. This procedure is repeated until a stop criteria is satisfied.

$$
P=\left[\begin{array}{cccc}
p_{1,1} & p_{1,2} & \cdots & p_{1, U} \\
p_{2,1} & p_{2,2} & \cdots & p_{2, U} \\
\vdots & \vdots & \ddots & \vdots \\
p_{n I n d, 1} & p_{n I n d, 2} & \cdots & p_{n I n d, U}
\end{array}\right]
$$

The crossover uses two breakpoints to generate a new chromosome, as shown in Fig. 6. In this figure, the chromosome $P_{z}$ is formed by the first series of $P_{x}$ chromosome, the second series of $P_{y}$ chromosome, and the third series of $P_{x}$ chromosome. Therefore, following the Fig. 4 , the GA metaheuristic in "Genetic Algorithm (I)" generates every unknowns of the $P$ population matrix by random uniform function. On the other hand, in "Genetic Algorithm (II)" and "Genetic Algorithm (III)" the initial population is fulfilled by best candidate solutions from previous GA executions, aiming to converge with lowest number iterations.

In order to illustrate this procedure and validate it, this work presents the optimization of a threedimensional photonic coupler for on-chip device. Its modeling is described in next section. The main optimization goal is to maximize the coupling interconnection between two optical waveguides with different dimensions (width and height) and electric properties. The first condition considers the output waveguide with high contrast of refractive index between core and substrate. The second condition is 


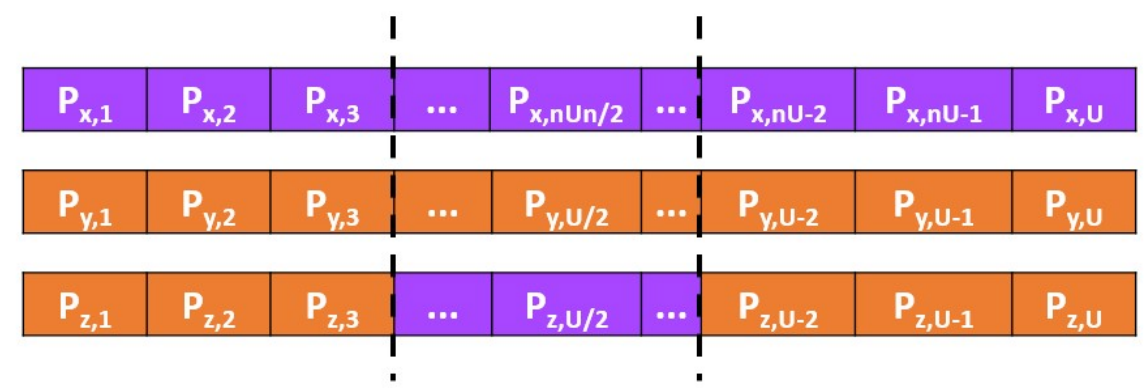

Fig. 6. Dual break point crossover operator of GA meta-heuristic.

associated to the reduced coupler geometry that makes it suitable to apply in high-density integration.

\section{NumERicAl RESULTS}

The proposed method, as already mentioned, was motivated by previous failed optimizations with GA considering every elements of the photonic coupler under the computational limitations, as described in the introduction section. Thereby, considering the multi-symmetric level (MSL) optimization herein presented, each GA execution was performed by 100 generations to iterate along their 150 chromosomes randomly matched by a recombination of $90 \%$ and similar mutation rate with single point uniform random function number generation, which is also applied to generate the gene value in the interval $[0,1]$. This limited number of generations for each GA (GA I, GA II and GA III) execution was considered to perform the simulations in a feasible runtime machine, where it was already mentioned in Section II, each 3D FEM execution requires around 114 seconds totalizing aproximatelly 6 days each parallel optimization execution with the cluster computer described.

The $P_{x, y}$ mutation value was a randomly generated by Scilabs' function rand, which values are between 0 to 1 . In order to associate $P_{x, y}$ to interesting refractive index, three different intervals values were set to associate them refractive index $n=1.0,1.98$, or 3.5. Therefore, when $0 \leqslant P_{x, y}<0.33$ to refractive index $n=1.0$, for $0.33 \leqslant P_{x, y}<0.66$ for $n=1.98$ and $n=3.5$ when $0.66 \leqslant P_{x, y} \leqslant 1.0$. These refractive indices were determined by the usual communication wavelength in $1.55 \mu \mathrm{m}$

Two different stop criteria were defined. At first, a minimal of $90 \%$ of coupling to stop the MSL iterative procedure. For example, if in the first level the optimization achieved coupling results above $90 \%$ the next symmetric optimizations were not executed. The second stop criteria is implicit to the GA metaheuristic such as already mentioned total of 100 iterations.

Therewith, the first MSL execution, considering the symmetry regions, was executed following the configurations described above and cross-section configuration is presented in Fig. 7. The coupling results were below 90\%, requiring the next MSL iteration level. Furthermore, it is shown in Fig. 7 distribution of the refractive indices in the frontal cross-section elements, being above the schematized the top-to-bottom view of these elements distributed in layers from 1 to 4 , respectively.

Brazilian Microwave and Optoelectronics Society-SBMO Brazilian Society of Electromagnetism-SBMag received 11 May 2021; for review 21 May 2021; accepted 10 Aug 2021 (C) 2021 SBMO/SBMag (cc) BY

ISSN 2179-1074 


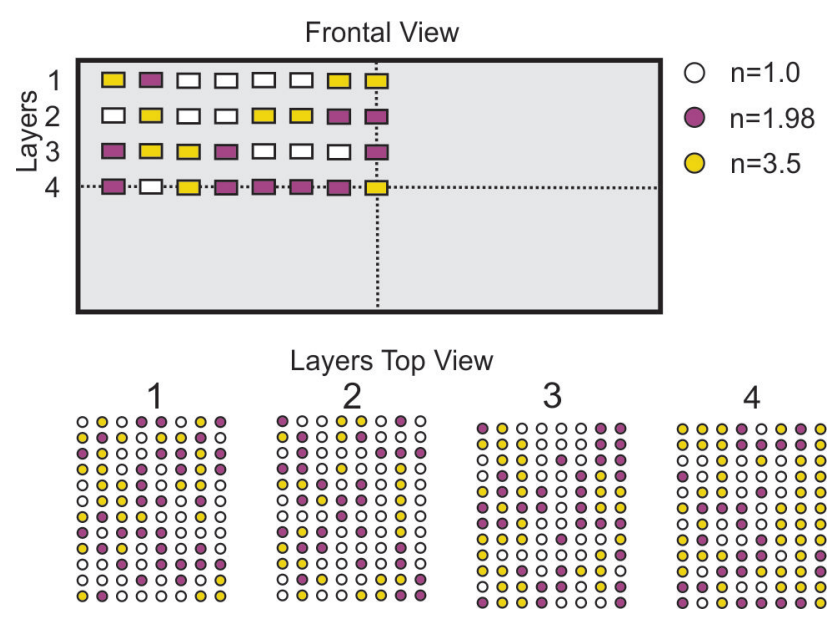

Fig. 7. First stage optimized optical coupler configuration.

The second MSL level considers the symmetry of the vertical geometries, making the total number of elements to be optimized to be half the number of cylinders in the optimization region. The Fig. 8 shows the frontal cross-section and top-to-bottom configuration of the cylindrical refractive indices.
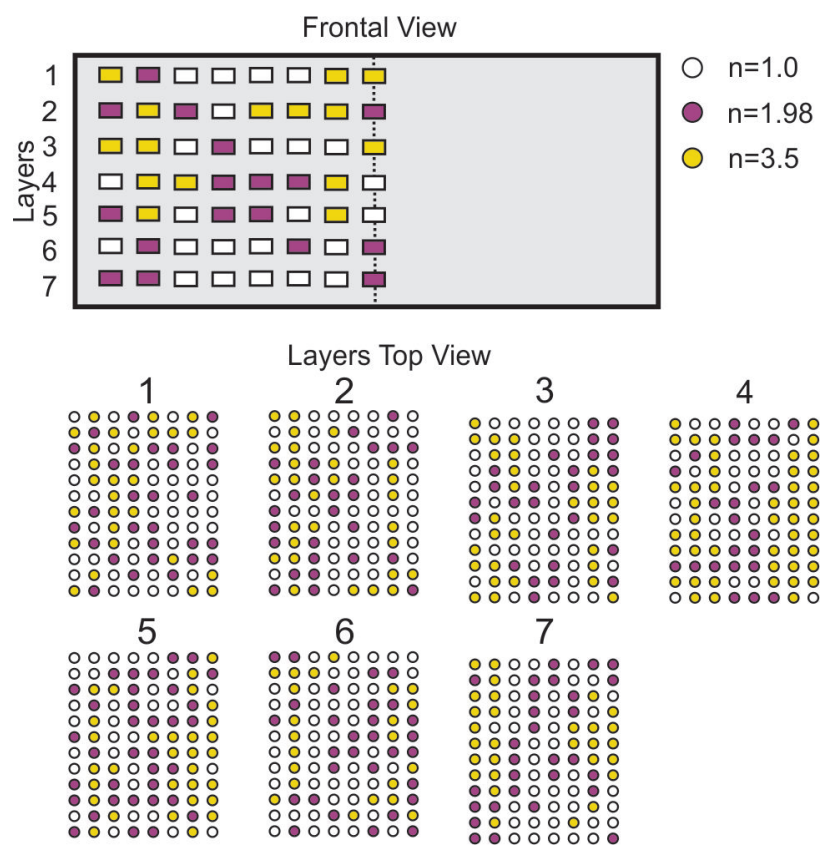

Fig. 8. Final optical coupler configuration.

This configuration resulted in a optical coupling around 94\%. The Fig. 9 shows the $x$-component of the electric field along the optical coupler and the transverse profile of this component in the output waveguide where it is possible to observe a delocalization of the mode from the waveguide's core due to the high refractive index. 


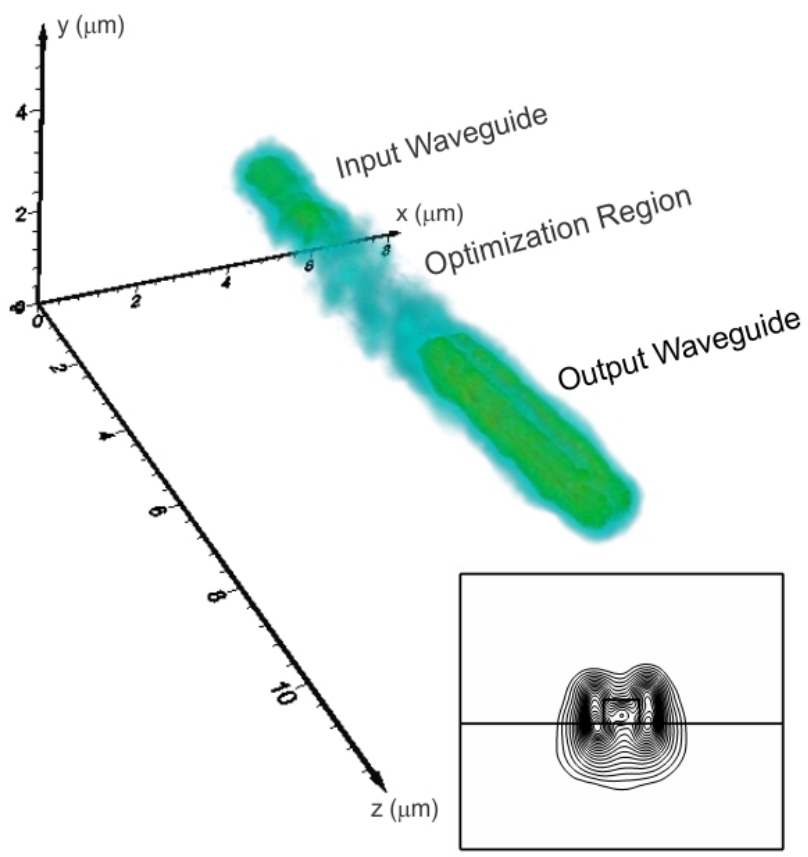

Fig. 9. The $x$-component of the electric field with an efficiency coupling of $94 \%$ and transverse profile of this component in the output waveguide.

Furthermore, in order to analyze the configuration limitation under different input wavelength, a narrow band from $1.5 \mu \mathrm{m}$ until $1.555 \mu \mathrm{m}$ was performed. The result is shown in Fig. 10 high coupling results are achieved by wavelengths greater than $1.533 \mu \mathrm{m}$. However, the performance of the coupler tends to decrease as wavelength moves away from this value.

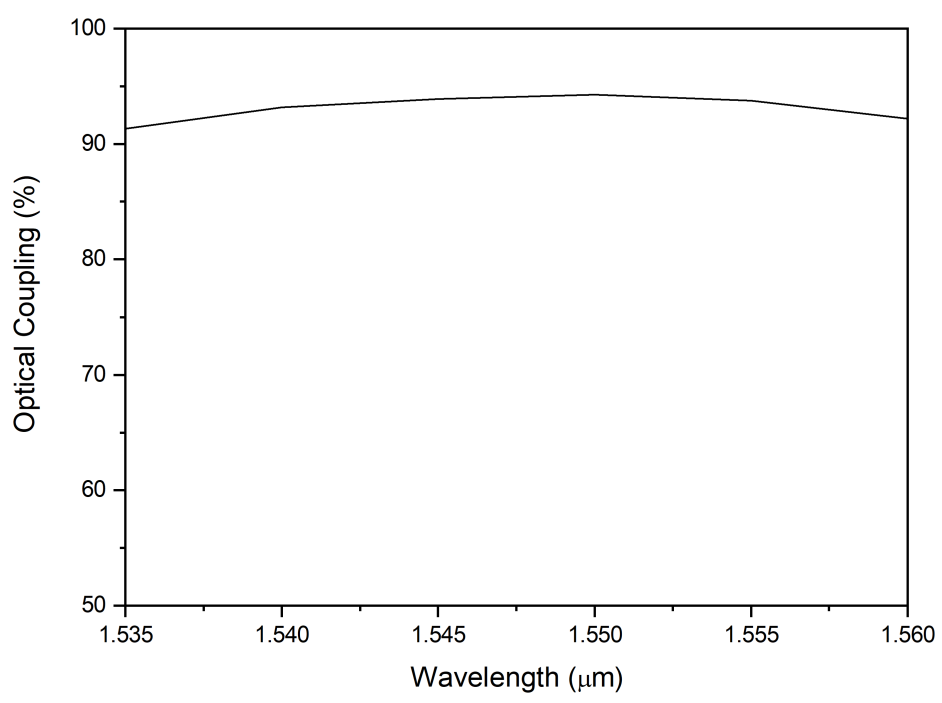

Fig. 10. Coupling efficiency as a function of wavelength.

\section{Conclusions}

The proposed MSL optimization procedure successfully achieved numerical results under the computational and physical-telecommunication imposed optimization conditions. The $94 \%$ coupling fee 
at the second from three optimization levels justify the successfully achievement of the proposed solution. These results attempt the initial computational conditions to overcome initial fail optimizations trials with GA and the same refractive indices 1.0, 198 and 3.5 to randomly select each of the 1260 unknowns. Furthermore, these numerical results presented a possibility to minimize photonic coupler devices considering forthcoming foundry technologies.

Finally, this work team group is currently working on novel optimizations procedures to achieve best results with minimal iterations by the integration of different metaheuristics (hybrid metaheuristics) and machine learning techniques.

\section{ACKNOWLEDGEMENTS}

The authors wish to thank the partial financial support by São Paulo Research Foundation (FAPESP) under the grant 2011/12792-3. They also thanks IFSP and Unicamp by technical support.

\section{REFERENCES}

[1] M. M. Shulaker, G. Hills, R. S. Park, R. T. Howe, K. Saraswat, H.-S. P. Wong, and S. Mitra, "Three-dimensional integration of nanotechnologies for computing and data storage on a single chip," Nature, vol. 547, no. 7661, pp. 74-78, 2017.

[2] S. Tomioka, A. Iguchi, and Y. Tsuji, "Function-expansion-based topology optimization of three-dimensional optical waveguide devices with multi-layered structure considering layer thickness," Optics Communications, p. 126094, 2020.

[3] Z. Cheng, J. Wang, Z. Yang, L. Zhu, Y. Yang, Y. Huang, and X. Ren, "Sub-wavelength grating assisted mode order converter on the soi substrate," Optics Express, vol. 27, no. 23, pp. 34 434-34 441, 2019.

[4] H. S. Dutta, A. K. Goyal, V. Srivastava, and S. Pal, "Coupling light in photonic crystal waveguides: A review," Photonics and Nanostructures-Fundamentals and Applications, vol. 20, pp. 41-58, 2016.

[5] E. Yablonovitch, "Photonic band structure: observation of an energy gap for light in 3-d periodic dielectric structures," in Quantum Electronics and Laser Science Conference, p. TUKK6, 1989.

[6] K. Berrada, "Geometric phase for a two-level system in photonic band gab crystal," Solid State Communications, vol. 273, pp. 34-38, 2018.

[7] R. K. Kushwaha, P. Karuppanan, and Y. Srivastava, "Proximity feed multiband patch antenna array with srr and pbg for thz applications," Optik, vol. 175, pp. 78-86, 2018.

[8] S. Ullah, C. Ruan, T. U. Haq, and X. Zhang, "High performance thz patch antenna using photonic band gap and defected ground structure," Journal of Electromagnetic Waves and Applications, vol. 33, no. 15, pp. 1943-1954, 2019.

[9] G. Á. Jiménez-Guzmán, J. A. Tirado-Mendez, A. Rangel-Merino, L. A. Vasquez-Toledo, and R. Marcelin-Jimenez, "Improving the performance of a patch antenna array by using photonic bandgap structures at x-band," Journal of Electromagnetic Waves and Applications, pp. 1-17, 2020.

[10] J.-S. Liu, J.-Y. Yang, H.-Y. Liu, and A.-D. Zhu, "Photon blockade by enhancing coupling via a nonlinear medium," Optics Express, vol. 28, no. 12, pp. 18397-18406, 2020.

[11] T. X. Hoang, S. T. Ha, Z. Pan, W. K. Phua, R. Paniagua-Dominguez, C. E. Png, H.-S. Chu, and A. I. Kuznetsov, "Collective mie resonances for directional on-chip nanolasers," Nano Letters, vol. 20, no. 8, pp. 5655-5661, 2020.

[12] M. H. Sani, A. Ghanbari, and H. Saghaei, "An ultra-narrowband all-optical filter based on the resonant cavities in rodbased photonic crystal microstructure," Optical and Quantum Electronics, vol. 52, p. 295, 2020.

[13] Y. Wang, S. Chen, P. P. Wen, S. Liu, and S. Zhong, "Multichannel filter based on tunable fibonacci quasi-periodic structure for one-dimensional magnetized ternary plasma photonic crystal," Optik, p. 165393, 2020.

[14] X. Zhang, "Time-dependent adjoint-based optimization of photonic crystals and metamaterials using a stabilized finite element method," 2017.

[15] M. Kadic, G. W. Milton, M. van Hecke, and M. Wegener, “3d metamaterials," Nature Reviews Physics, vol. 1, no. 3, pp. 198-210, 2019.

[16] T. Sugie, T. Akamatsu, T. Nishitsuji, R. Hirayama, N. Masuda, H. Nakayama, Y. Ichihashi, A. Shiraki, M. Oikawa, N. Takada, et al., "High-performance parallel computing for next-generation holographic imaging," Nature Electronics, vol. 1, no. 4, pp. 254-259, 2018.

[17] K. Aydin, "Nanostructured silicon success," Nature Photonics, vol. 9, no. 6, pp. 353-355, 2015.

Brazilian Microwave and Optoelectronics Society-SBMO

Brazilian Society of Electromagnetism-SBMag received 11 May 2021; for review 21 May 2021; accepted 10 Aug 2021 (C) 2021 SBMO/SBMag (cc) BY

ISSN 2179-1074 
[18] Y. Yang, Z. Gao, H. Xue, L. Zhang, M. He, Z. Yang, R. Singh, Y. Chong, B. Zhang, and H. Chen, "Realization of a three-dimensional photonic topological insulator," Nature, vol. 565, no. 7741, pp. 622-626, 2019.

[19] K. Yao, R. Unni, and Y. Zheng, "Intelligent nanophotonics: merging photonics and artificial intelligence at the nanoscale," Nanophotonics, vol. 8, no. 3, pp. 339-366, 2019.

[20] M. D. Huntington, L. J. Lauhon, and T. W. Odom, "Subwavelength lattice optics by evolutionary design," Nano letters, vol. 14, no. 12, pp. 7195-7200, 2014.

[21] F. Meng, X. Huang, and B. Jia, "Bi-directional evolutionary optimization for photonic band gap structures," Journal of Computational Physics, vol. 302, pp. 393-404, 2015.

[22] G. N. Malheiros-Silveira and F. G. Delalibera, "Inverse design of photonic structures using an artificial bee colony algorithm," Applied Optics, vol. 59, no. 13, pp. 4171-4175, 2020.

[23] T. Feichtner, O. Selig, M. Kiunke, and B. Hecht, "Evolutionary optimization of optical antennas," Physical review letters, vol. 109, no. 12, p. 127701, 2012.

[24] C. H. da Silva Santos, M. S. Goncalves, and H. E. Hernandez-Figueroa, "Designing novel photonic devices by bioinspired computing," IEEE Photonics Technology Letters, vol. 22, no. 15, pp. 1177-1179, 2010.

[25] A. da Silva Ferreira, C. H. da Silva Santos, M. S. Gonçalves, and H. E. H. Figueroa, "Towards an integrated evolutionary strategy and artificial neural network computational tool for designing photonic coupler devices," Applied Soft Computing, vol. 65, pp. 1-11, 2018.

[26] A. Dourado-Sisnando, V. F. Rodríguez-Esquerre, C. Rubio-Mercedes, L. d. F. Vieira, and I. M. Ruffini, "Power coupling optimization in $2 \mathrm{~d}$ waveguides by evolutionary algorithms," IEEE Photonics Technology Letters, vol. 27, no. 14, pp. 1561-1564, 2015.

[27] H. Ghorbaninejad and A. Ghajar, "Genetic algorithm design for e-plane waveguide filters," Optical and Quantum Electronics, vol. 48, no. 10, p. 468, 2016.

[28] M. Mohamadrezaee, M. Shirali, M. Akhlaghi, and M. Kaboli, "Combination of binary particle swarm optimization algorithm and discrete dipole approximation method to investigate the plasmonic circuit-based coherent perfect absorption filter," Optical and Quantum Electronics, vol. 49, no. 3, p. 100, 2017.

[29] J. L. Bentley, "Multidimensional divide-and-conquer," Communications of the ACM, vol. 23, no. 4, pp. 214-229, 1980.

[30] Y. Ishizaka, Y. Kawaguchi, K. Saitoh, and M. Koshiba, "Three-dimensional finite-element solutions for crossing slotwaveguides with finite core-height," Journal of lightwave technology, vol. 30, no. 21, pp. 3394-3400, 2012.

[31] J. Jian-Ming, "The finite element method in electromagnetics. 2002."

[32] Y. Tsuji and M. Koshiba, "Finite element beam propagation method with perfectly matched layer boundary conditions for three-dimensional optical waveguides," International Journal of Numerical Modelling: Electronic Networks, Devices and Fields, vol. 13, no. 2-3, pp. 115-126, 2000.

[33] M. Mitchell, An introduction to genetic algorithms. MIT press, 1998.

[34] A. Bajaj and O. P. Sangwan, "A systematic literature review of test case prioritization using genetic algorithms," IEEE Access, vol. 7, pp. 126355-126375, 2019. 